\title{
Susceptibilidad antimicrobiana de cepas de Pseudomonas aeruginosa aisladas en el laboratorio del Hospital Regional Dr. Leonardo Guzmán de Antofagasta, Chile
}

\author{
ALCIDES ZAMBRANO F. y NELSON HERRERA A.
}

\author{
In vitro antimicrobial susceptibility of Pseudomonas aeruginosa strains \\ isolated at Hospital Dr. Leonardo Guzmán, Antofagasta, Chile
}

Pseudomonas aeruginosa is a nosocomial pathogen that often displays a high degree of antibiotic resistance. This pathogen causes also serious infections specially in patients with severe diseases or immunodeficiency. To offer the best treatment in every institution it is necessary to know the local pattern of antimicrobial susceptibility, then we studied the antibiotic susceptibility of $P$. aeruginosa strains isolated from patients attended in the Regional Hospital of Antofagasta. Most of them had an underlying disease that predisposed them to the infection and $48 \%$ had a severe infection. The strains showed higher drug resistance than that reported by other chilean researchers. $P$. aeruginosa displayed high resistance to amikacin $(36,8 \%)$, ceftazidime $(36,8 \%)$ and ciprofloxacin $(68,4 \%)$ intermediate resistance to imipenem $(26,3 \%)$, but low resistance to piperacillin/tazobactam $(5,3 \%)$ and cefoperazone/sulbactam (15,8\%). This is the first drug susceptibility study conducted in the Second Region of Chile, where $P$. aeruginosa was assayed against those antibiotics used in the clinical practice.

Key words: Pseudomonas aeruginosa, Drug susceptibility; Carbapenems; Aminoglycosides.

Palabras claves: Pseudomonas aeruginosa; Susceptibilidad antimicrobiana; Carbapenems; Aminoglucósidos.

\section{Introducción}

Pseudomonas aeruginosa es una de las bacterias gramnegativas más comúnmente aisladas en infecciones nosocomiales, especialmente en unidades de cuidados intensivos ${ }^{1-6}$. De acuerdo a cifras del CDC, la incidencia de infecciones por $P$. aeruginosa en hospitales de Estados Unidos de Norteamérica alcanza a $0,4 \%$ de los egresos totales y provoca $10,1 \%$ de todas las infecciones nosocomiales ${ }^{7}$. Es por ello reportada como el agente etiológico de $16 \%$ de las neumonías, $12 \%$ de las infecciones del tracto urinario y $8 \%$ de las infecciones postquirúrgicas ${ }^{8}$. Según datos del MINSAL, en Chile $P$. aeruginosa es el microorganismo más frecuentemente aislado en neumo- nías nosocomiales asociadas a ventilación mecánica en UCIs pediátricas, 30,6\%, y el tercero en unidades de cuidados intensivos de adultos, donde representa $18,5 \%$ de los casos $^{6}$. Es también un importante agente causal de infecciones del tracto urinario en servicios quirúrgicos, de medicina interna y en UCIs ${ }^{6}$.

Las infecciones por $P$. aeruginosa rara vez son adquiridas en la comunidad por pacientes inmunocompetentes; sin embargo, cuando se alteran las barreras normales de la piel y mucosas (heridas, quemaduras, intubación endotraqueal, cateterismo vesical, vías venosas), frente a estados de inmunodepresión (senilidad, diabetes mellitus, cáncer, SIDA, neutropenia), se reduce la flora bacteriana intestinal que ejerce un efecto

Unidad de Cuidados Intensivos, Hospital “Dr. Leonardo Guzmán”, Antofagasta (AZF).

Unidad de Microbiología, Facultad de Ciencias de la Salud, Universidad de Antofagasta (NHA).

Recibido: 15 octubre 2003

Aceptado: 6 marzo 2004 
protector por el uso de antimicrobianos de amplio espectro, o el paciente es expuesto a reservorios del ambiente hospitalario, puede actuar como patógeno primario ${ }^{8-11}$. Bajo estas circunstancias, $P$. aeruginosa puede provocar infecciones graves como: bacteriemias, neumonía, infecciones del SNC, infecciones del tracto urinario e infecciones cutáneas en grandes quemados ${ }^{8,12}$. Estas infecciones, generalmente nosocomiales, tienen un curso fulminante y una letalidad extremadamente alta a pesar de un tratamiento antimicrobiano adecuado ${ }^{12-14}$. Este microorganismo también puede causar infecciones de otros sistemas, como: otitis externas y otitis supurativa crónica, queratitis y úlceras corneales, endoftalmitis, artritis séptica y osteomielitis, infecciones gastrointestinales, ectima gangrenoso, infección de úlceras y escaras cutáneas ${ }^{12}$. Estas últimas no significan una amenaza inmediata para la vida del paciente, pero pueden adquirir un curso crónico, en el cual los microorganismos patógenos suelen ser difíciles de erradicar ${ }^{12}$.

$P$. aeruginosa es naturalmente resistente a muchos de los antimicrobianos de uso habitual en la práctica clínica, debido a la barrera de permeabilidad ofrecida por su membrana externa de LPS y a plasmidios de resistencia antimicrobiana, entre otros factores ${ }^{11}$. Las infecciones graves y nosocomiales por $P$. aeruginosa requieren generalmente un tratamiento antimicrobiano asociado con el fin de lograr un mayor efecto bactericida y reducir la aparición de resistencia a ellos $^{12,13}$. Los antimicrobianos con efecto anti pseudomonas comprenden aminoglucósidos (amikacina, gentamicina), cefalosporinas de $3^{\text {a }}$ (ceftazidima, cefoperazona) y $4^{a}$ generación (cefepime), monobactámicos (aztreonam), carbapenems (imipenem, meropenem), fluoroquinolonas (ciprofloxacina) y penicilinas de espectro ampliado (ticarcilina, carbenicilina, ticarcilina/ácido clavulánico, piperacilina, piperacilina/tazobactam, mezlocilina). Los patrones locales de susceptibilidad deben considerarse en la elección inicial del antimicrobiano, mientras que el estudio de la susceptibilidad de la cepa aislada del enfermo orienta el tratamiento antimicrobiano definitivo ${ }^{14-17}$.

El propósito de este estudio fue determinar la susceptibilidad antimicrobiana de cepas de $P$. aeruginosa aisladas desde pacientes atendidos en diferentes servicios clínicos del Hospital Regional "Dr. Leonardo Guzmán" de Antofagasta.

\section{Material y Métodos}

Las cepas de $P$. aeruginosa se recolectaron durante el primer semestre del año 2002, desde
19 pacientes pediátricos y adultos hospitalizados en diferentes servicios clínicos: Medicina, Unidad de Tratamientos Intermedios (UTIM), Cirugía, Servicio de Atención Intermedia Quirúrgica (SAIQ), UCI de adultos (UCI-A), Pediatría, UCI pediátrica (UCI-P) y Neonatología. Estas correspondieron a la totalidad de cepas de $P$. aeruginosa, identificadas desde pacientes hospitalizados, durante ese período en el laboratorio del Hospital Regional de Antofagasta. Se consideró un solo aislamiento de $P$. aeruginosa por paciente.

Para su estudio e identificación las cepas se sembraron en agar sangre y agar MacConkey, incubándose durante $24 \mathrm{hr}$ a $37^{\circ} \mathrm{C}$. Posteriormente, se observaron las características macroscópicas de las colonias (forma, tamaño, producción de pigmento, olor). A aquellas colonias que no fermentaban lactosa, se les sometió a pruebas bioquímicas, según pautas del Instituto de Salud Pública de Chile ${ }^{18}$, y a cultivo en medios King A y King B, durante 24 horas a $37^{\circ} \mathrm{C}$.

El estudio de la susceptibilidad antimicrobiana de las cepas se realizó mediante el método cuantitativo de dilución seriada en agar según Ericcson y Sherris ${ }^{19}$, utilizado como referente por el NCCLS $^{20}$. Brevemente, el método de Ericcson y Sherris consiste en cultivar cada cepa a evaluar en placas de agar Mueller Hinton, conteniendo diferentes concentraciones previamente establecidas de un antibacteriano determinado. Se observó luego de 18 horas el desarrollo bacteriano a las diferentes diluciones de cada antimicrobiano.

En nuestro ensayo se probaron ocho antimicrobianos, siendo todos evaluados a once concentraciones, en el rango de $0,25 \mu \mathrm{g} / \mathrm{ml}$ a $256 \mu \mathrm{g} / \mathrm{ml}$, según recomendaciones del NCCLS ${ }^{20}$. Las diluciones de los antimicrobianos a ser evaluados fueron preparadas a partir de los fármacos puros, obtenidas como se detalla a continuación: imipenem (Merck Sharp \& Dohme), piperacilina/ tazobactam (Wyeth), cefoperazona/sulbactam (Pfizer), ceftazidima (GlaxoSmithKline), amikacina (Sigma-Aldrich, USA), gentamicina (SigmaAldrich, USA), ciprofloxacina (Bayer), levofloxacina (Aventis Pharma).

La CIM se definió como la menor concentración del antimicrobiano capaz de inhibir el crecimiento del inóculo de la cepa en el medio de cultivo.

Las cepas fueron consideradas susceptibles (S), de susceptibilidad intermedia (I) o resistentes (R), de acuerdo a patrones de interpretación de CIM internacionales (NCCLS) ${ }^{21}$. La CIM para cefoperazona/sulbactam fue interpretada de acuerdo a recomendaciones del fabicante, validadas en la literatura ${ }^{22,23}$. Los puntos de corte de CIM $(\mu \mathrm{g} / \mathrm{ml})$ 
fueron los siguientes: imipenem $\mathrm{S}<4, \mathrm{I}=8, \mathrm{R}>16$; piperacilina/tazobactam $\mathrm{S}<64 / 4, \mathrm{R}>128 / 4$; cefoperazona/sulbactam $\mathrm{S}<16 / 8, \mathrm{I}=32 / 16$, $\mathrm{R}>64 / 32$; ceftazidima $\mathrm{S}<8, \mathrm{I}=16, \mathrm{R}>32$; amikacina $\mathrm{S}<16, \mathrm{I}=32, \mathrm{R}>64$; gentamicina $\mathrm{S}<4, \mathrm{I}=8, \mathrm{R}>16$; ciprofloxacina $\mathrm{S}<1, \mathrm{I}=2$, $\mathrm{R}>4$; levofloxacina $\mathrm{S}<2, \mathrm{I}=4, \mathrm{R}>8^{21,22}$.

Como control de calidad se utilizó la cepa de referencia $P$. aeruginosa $\mathrm{ATCC}^{\circledR} 27853$. Se realizó control de calidad para todos los antimicrobianos, considerando como aceptables los siguientes rangos de CIM ( $\mu \mathrm{g} / \mathrm{ml})$ : imipenem 1-4, piperacilina/tazobactam 1/4-8/4, cefoperazona/ sulbactam 2/1-8/4, ceftazidima 1-4, amikacina 1-4, gentamicina 0,5-2, ciprofloxacina 0,25-1, levofloxacina $0,5-4^{22,24}$. Todos los controles estuvieron en el rango aceptable.

La información requerida sobre los pacientes se obtuvo desde sus fichas clínicas.

\section{Resultados}

Las cepas de $P$. aeruginosa se aislaron desde 19 pacientes hospitalizados. Las características de los pacientes se muestran en la Tabla 1. La mayoría era de sexo masculino y el rango de edad oscilaba entre 13 días y 84 años, siendo el promedio de 41 años. La mayoría (17 pacientes) tenía alguna condición que alteraba los mecanismos defensivos normales o una hospitalización prolongada, que predisponía a la infección con $P$. aeruginosa. De los factores predisponentes a infecciones por $P$. aeruginosa (relacionados con el sitio de la infección) los más frecuentes eran lesiones o heridas cutáneas, traqueostomía, cáncer y diabetes mellitus. Doce pacientes tenían una infección grave con riesgo vital $(63,2 \%)$. La localización más frecuente de la infección fue la vía respiratoria, aislándose $P$. aeruginosa desde la expectoración o aspirado traqueal en 4 pacientes $(21 \%)$.

Quince pacientes $(78,9 \%)$ tenían una infección nosocomial, entendiéndose como tal aquella infección que aparece después de las 48 hrs de estadía hospitalaria, y ésta afectaba en mayor número a la piel. El grupo de pacientes con infección nosocomial tenía un promedio de días de hospitalización mayor (52,4 versus 15 días en el grupo con infección extrahospitalaria).

La procedencia de las 19 cepas de P. aeruginosa aisladas durante el período de recolección en el laboratorio del Hospital Regional de Antofagasta y los resultados de susceptibilidad in vitro se muestran en las Tablas 2 y 3 respectivamente. Las cepas aisladas de las diferentes mues- tras estudiadas fueron susceptibles a los antimicrobianos en distinta medida (Tablas 4 y 5). De las quinolonas, ciprofloxacina presentó una resistencia $(68,4 \%)$ levemente inferior a levofloxacina $(78,9 \%)$. En el grupo de aminoglicósidos, llamó la atención la menor resistencia de las cepas a gentamicina que a amikacina (26,3 versus $36,8 \%$, respectivamente). Entre las cefalosporinas, ceftazidima mostró resistencia más alta que la combinación de cefoperazona/sulbactam ( 36,8 versus $15,8 \%$, respectivamente). Para imipenem hubo $26,3 \%$ de resistencia, mientras que la penicilina con acción antipseudomonas, piperacilina, en combinación con tazobactam, mostró el índice más bajo de resistencia con sólo $5,3 \%$.

Se observaron cepas susceptibles a la mayoría de los antimicrobianos ensayados, en cambio otras presentaron escasa sensibilidad, encontrándose además cepas con antibiotipos semejantes (Tabla 6).

Tabla 1. Características de los 19 pacientes hospitalizados en que se aisló $P$. aeruginosa

Edad Promedio 41,0 años (13 días a 84 años)

Sexo Masculino $12 \quad$ Femenino 7

Condición predisponente: $\quad \mathbf{N}^{\circ}$ pacientes

$\begin{array}{ll}\text { Cáncer } & 3 \\ \text { Diabetes mellitus } & 2 \\ \text { Traqueostomía } & 2 \\ \text { Herida operatoria } & 2 \\ \text { Catéter vesical } & 1 \\ \text { Cistostomía } & 1 \\ \text { Fibrosis quística } & 1 \\ \text { Herida de piel } & 1 \\ \text { Insuficiencia renal crónica } & 1 \\ \text { Prematurez extrema } & 1 \\ \text { Quemaduras } & 1 \\ \text { SIDA } & 1 \\ \text { Tubo pleural } & 1\end{array}$

Días hospitalización: Promedio 42,5 días

Rango 3 a 128 días

Infección nosocomial: 15 pacientes 
Tabla 2. Origen de las 19 cepas de Pseudomonas aeruginosa aisladas en el Hospital Regional Dr. Leonardo Guzmán de Antofagasta, Chile

\begin{tabular}{|c|c|c|c|c|c|c|c|c|c|c|c|}
\hline $\begin{array}{l}\text { Servicio } \\
\text { Clínico }\end{array}$ & Sangre & $\begin{array}{l}\text { Secreción } \\
\text { traqueal, } \\
\text { expectoración }\end{array}$ & Orina & $\begin{array}{c}\text { Deposi- } \\
\text { ciones }\end{array}$ & $\begin{array}{l}\text { Lesión } \\
\text { cutánea }\end{array}$ & $\begin{array}{l}\text { Quema- } \\
\text { dura }\end{array}$ & $\begin{array}{l}\text { Fístula } \\
\text { LCR }\end{array}$ & $\begin{array}{c}\text { Líquido } \\
\text { peritoneal }\end{array}$ & $\begin{array}{c}\text { Secreción } \\
\text { uretral }\end{array}$ & $\begin{array}{l}\text { Drenaje } \\
\text { pleural }\end{array}$ & $\begin{array}{l}\text { Prótesis } \\
\text { vascular }\end{array}$ \\
\hline Medicina & & & 1 & & & & & & & & \\
\hline Cirugía & & 1 & & 1 & 1 & & & & & 1 & 1 \\
\hline Pediatría & 1 & 2 & & & & & & 1 & & & \\
\hline TIM & & & & & 1 & & & & 1 & & \\
\hline SAIQ & & & & & 1 & 1 & & & & & \\
\hline Neonatología & & & & 1 & & & 1 & 1 & & & \\
\hline UCI A & & 1 & & & & & & & & & \\
\hline UCI P & & & & 1 & & & & & & & \\
\hline Total & 1 & 4 & 1 & 3 & 3 & 1 & 1 & 2 & 1 & 1 & 1 \\
\hline
\end{tabular}

TIM = Unidad de tratamiento intermedio médico

SAIQ = Servicio de atención intermedia quirúrgico

UCI A = Unidad de cuidados intensivos adultos

UCI P = Unidad de cuidados intensivos pediátricos

Tabla 3. Susceptibilidad de 19 cepas de Pseudomonas aeruginosa frente a diferentes antimicrobianos. Antofagasta, Chile

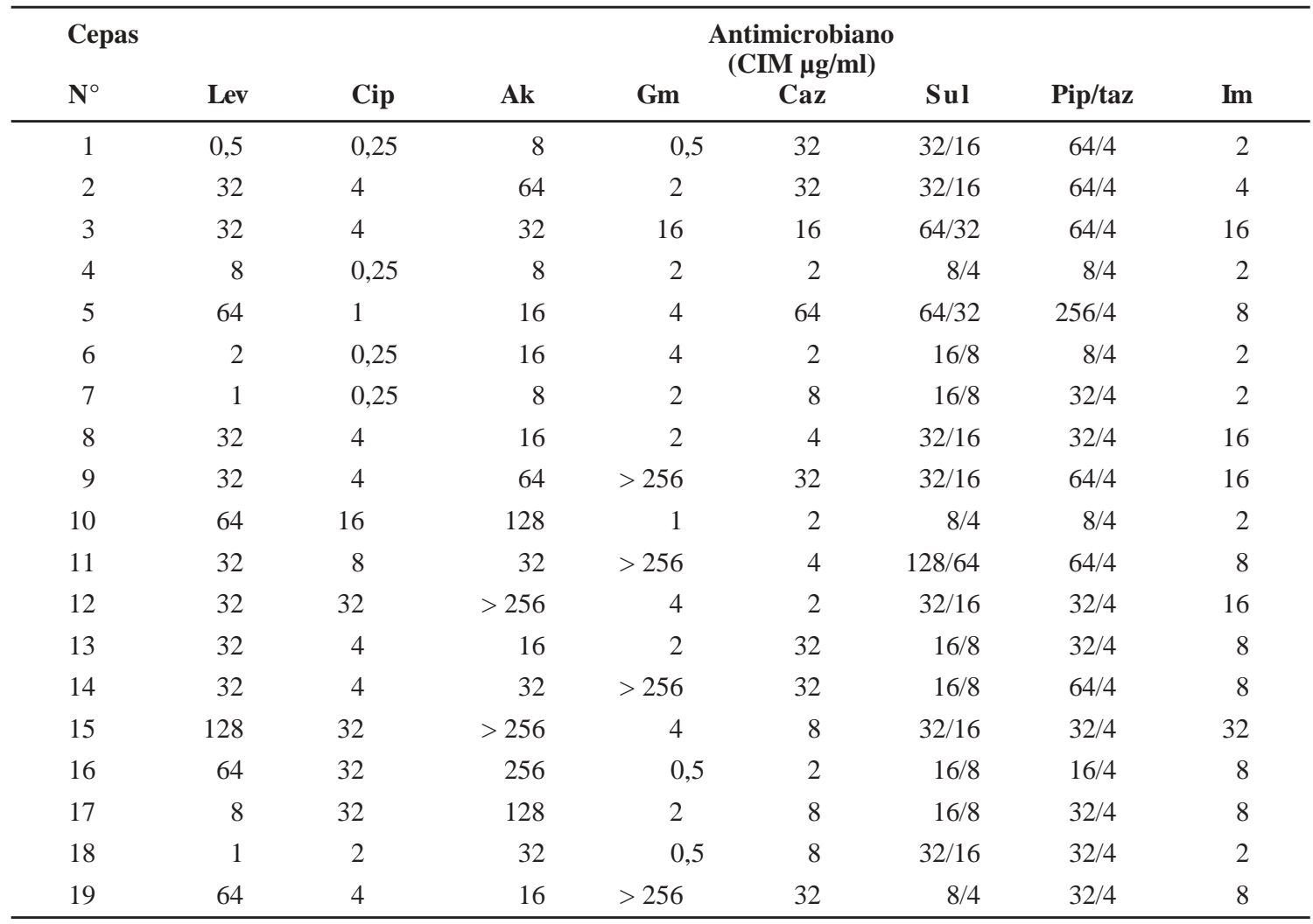

Lev = levofloxacina

Cip = ciprofloxacina

$\mathrm{Ak}=$ amikacina
$\mathrm{Gm}=$ gentamicina

$\mathrm{Caz}=$ ceftazidima

Sul = cefoperazona/sulbactam
Pip/taz = piperacilina/tazobactam $\mathrm{Im}=$ imipenem 
Tabla 4. Porcentajes de susceptibilidad de las cepas de Pseudomonas aeruginosa frente a 8 antimicrobianos

\begin{tabular}{|c|c|c|c|c|c|c|}
\hline \multirow[t]{2}{*}{ Antimicrobiano } & \multicolumn{2}{|c|}{ Susceptibles } & \multicolumn{2}{|c|}{$\begin{array}{c}\text { Cepas } \\
\text { Susceptibilidad } \\
\text { intermedia }\end{array}$} & \multicolumn{2}{|c|}{ Resistentes } \\
\hline & $\mathbf{N}^{\circ}$ & $\%$ & $\mathbf{N}^{\circ}$ & $\%$ & $\mathbf{N}^{\circ}$ & $\%$ \\
\hline Levofloxacina & 4 & 21,1 & -- & -- & 15 & 78,9 \\
\hline Ciprofloxacina & 5 & 26,3 & 1 & 5,3 & 13 & 68,4 \\
\hline Amikacina & 8 & 42,1 & 4 & 21,1 & 7 & 36,8 \\
\hline Gentamicina & 14 & 73,7 & -- & -- & 5 & 26,3 \\
\hline Ceftazidima & 11 & 57,9 & 1 & 5,3 & 7 & 36,8 \\
\hline Cefoperazona/sulbactam & 9 & 47,4 & 7 & 36,8 & 3 & 15,8 \\
\hline Piperacilina/tazobactam & 18 & 94,7 & -- & -- & 1 & 5,3 \\
\hline Imipenem & 7 & 36,8 & 7 & 36,8 & 5 & 26,3 \\
\hline
\end{tabular}

Tabla 5. Porcentajes de susceptibilidad de las cepas de Pseudomonas aeruginosa a diferentes concentraciones de antimicrobianos

\begin{tabular}{|c|c|c|c|c|c|c|c|c|c|c|c|c|c|c|c|c|}
\hline \multirow[t]{3}{*}{ Antimicrobiano } & \multicolumn{16}{|c|}{ CIM $(\mu \mathrm{g} / \mathrm{ml})$} \\
\hline & \multicolumn{2}{|c|}{$<1$} & \multicolumn{2}{|c|}{2} & \multicolumn{2}{|c|}{4} & \multicolumn{2}{|c|}{8} & \multicolumn{2}{|c|}{16} & \multicolumn{2}{|c|}{32} & \multicolumn{2}{|c|}{64} & \multicolumn{2}{|c|}{$>128$} \\
\hline & $\mathbf{N}^{\circ}$ & $\%$ & $\mathbf{N}^{\circ}$ & $\%$ & $\mathbf{N}^{\circ}$ & $\%$ & $\mathbf{N}^{\circ}$ & $\%$ & $\mathbf{N}^{\circ}$ & $\%$ & $\mathbf{N}^{\circ}$ & $\%$ & $\mathbf{N}^{\circ}$ & $\%$ & $\mathbf{N}^{\circ}$ & $\%$ \\
\hline Levofloxacina & 3 & 15,8 & 1 & 5,3 & & & 2 & 10,5 & & & 8 & 42,1 & 4 & 21,1 & 1 & 5,3 \\
\hline Ciprofloxacina & 5 & 26,3 & 1 & 5,3 & 7 & 36,8 & 1 & 5,3 & 1 & 5,3 & 4 & 21,1 & & & & \\
\hline Amikacina & & & & & & & 3 & 15,8 & 5 & 26,3 & 4 & 21,1 & 2 & 10,5 & 5 & 26,3 \\
\hline Gentamicina & 4 & 21,1 & 6 & 31,5 & 4 & 21,1 & & & 1 & 5,3 & & & & & 4 & 21,1 \\
\hline Ceftazidima & & & 5 & 26,3 & 2 & 10,5 & 4 & 21,1 & 1 & 5,3 & 6 & 31,5 & 1 & 5,3 & & \\
\hline Cefoperazona /sulbactam & & & & & & & 3 & 15,8 & 6 & 31,5 & 7 & 36,8 & 2 & 10,5 & 1 & 5,3 \\
\hline Piperacilina /tazobactam & & & & & & & 3 & 15,8 & 1 & 5,3 & 8 & 42,1 & 6 & 31,5 & 1 & 5,3 \\
\hline Imipenem & & & 6 & 31,5 & 1 & 5,3 & 7 & 36,8 & 4 & 21,1 & 1 & 5,3 & & & & \\
\hline
\end{tabular}

Tabla 6. Perfil de susceptibilidad a antimicrobianos de las 19 cepas de Pseudomonas aeruginosa

\begin{tabular}{cl}
\hline Cepas & Susceptibilidad a antimicrobianos* \\
\hline 1 & $\mathrm{Lev}-\mathrm{Gm}-\mathrm{Ak}-\mathrm{Im}-\mathrm{Cip}-\mathrm{Caz}-\mathrm{Sul}-\mathrm{Pip} / \mathrm{taz}$ \\
2 & $\mathrm{Lev}-\mathrm{Gm}-\mathrm{Ak}-\mathrm{Im}-\mathrm{Cip}-\mathrm{Caz}-\mathrm{Sul}-\mathrm{Pip} / \mathrm{taz}$ \\
3 & $\mathrm{Lev}-\mathrm{Gm}-\mathrm{Ak}-\mathrm{Im}-\mathrm{Cip}-\mathrm{Caz}-\mathrm{Sul}-\mathrm{Pip} / \mathrm{taz}$ \\
4 & $\mathrm{Lev}-\mathrm{Gm}-\mathrm{Ak}-\mathrm{Im}-\mathrm{Cip}-\mathrm{Sul}-\mathrm{Pip} / \mathrm{taz}$ \\
5 & $\mathrm{Gm}-\mathrm{Im}-\mathrm{Ak}-\mathrm{Cip}-\mathrm{Caz}-\mathrm{Sul}-\mathrm{Pip} / \mathrm{taz}$ \\
6 & $\mathrm{Gm}-\mathrm{Im}-\mathrm{Caz}-\mathrm{Sul}-\mathrm{Pip} / \mathrm{taz}$ \\
7 & $\mathrm{Gm}-\mathrm{Im}-\mathrm{Caz}-\mathrm{Sul}-\mathrm{Pip} / \mathrm{taz}$ \\
8 & $\mathrm{Gm}-\mathrm{Im}-\mathrm{Caz}-\mathrm{Sul}-\mathrm{Pip} / \mathrm{taz}$ \\
9 & $\mathrm{Gm}-\mathrm{Ak}-\mathrm{Caz}-\mathrm{Sul}-\mathrm{Pip} / \mathrm{taz}$ \\
10 & $\mathrm{Gm}-\mathrm{Ak}-\mathrm{Im}-\mathrm{Sul}-\mathrm{Pip} / \mathrm{taz}$ \\
11 & $\mathrm{Ak}-\mathrm{Im}-\mathrm{Sul}-\mathrm{Pip} / \mathrm{taz}$ \\
12 & $\mathrm{Ak}-\mathrm{Im}-\mathrm{Sul}-\mathrm{Pip} / \mathrm{taz}$ \\
13 & $\mathrm{Gm}-\mathrm{Caz}-\mathrm{Sul}-\mathrm{Pip} / \mathrm{taz}$ \\
14 & $\mathrm{Gm}-\mathrm{Caz}-\mathrm{Sul}-\mathrm{Pip} / \mathrm{taz}$ \\
15 & $\mathrm{Gm}-\mathrm{Im}-\mathrm{Sul}-\mathrm{Pip} / \mathrm{taz}$ \\
16 & $\mathrm{Gm}-\mathrm{Ak}-\mathrm{Im}-\mathrm{Cip}$ \\
17 & $\mathrm{Ak}-\mathrm{Im}-\mathrm{Caz}-\mathrm{Pip} / \mathrm{taz}$ \\
18 & $\mathrm{Ak}-\mathrm{Caz}-\mathrm{Pip} / \mathrm{taz}$ \\
19 & $\mathrm{Cip}-\mathrm{Sul}-\mathrm{Pip} / \mathrm{taz}$ \\
\hline
\end{tabular}

*Códigos en Tabla 3

\section{Discusión}

Pseudomonas aeruginosa puede causar infecciones nosocomiales graves, las cuales tienen una alta letalidad ${ }^{25,26,36,38}$. En este contexto, el incremento emergente de cepas resistentes a antimicrobianos es un problema mayor en la práctica clínica ${ }^{32,33,39}$. Dentro de los mecanismos de resistencia a los antimicrobianos se incluyen: capacidad de formar biopelículas, gracias a la producción de alginato (ej: resistencia a $\beta$-lactámicos, aminoglucósidos, fluoroquinolonas); disminución de la permeabilidad de la membrana externa; bombas de eflujo para multiples fármacos (aminoglucósidos, imipenem, tetraciclina, fluoroquinolonas $)^{37}$ y enzimas que modifican los antibacterianos ( $\beta$-lactámicos, aminoglucósidos). De ahí la importancia de conocer los patrones de susceptibilidad locales de $P$. aeruginosa ${ }^{15,16,27,28,31,35}$.

En este estudio las infecciones nosocomiales por $P$. aeruginosa se asociaron a una hospitalización más prolongada, lo cual está en concordancia con lo descrito en la literatura ${ }^{8,26}$. Lo anterior puede explicarse porque la mayor estadía en un 
ambiente hospitalario prolonga la exposición del paciente a microorganismos como $P$. aeruginosa $\mathrm{y}$, por otro lado, la intercurrencia de una infección intrahospitalaria obliga a la mantención de la hospitalización hasta su resolución. Además del empeoramiento de la salud de un paciente, se debe considerar el efecto económico de una infección nosocomial, pues prolonga la hospitalización, aumenta el gasto en antimicrobianos y de otras medidas terapéuticas, lo cual encarece los $\operatorname{costos}^{26,29,30,39}$. Para tratar de controlar este problema se han creado los Comités de Infecciones Intrahospitalarias ${ }^{6}$, instancias que se preocupan de implementar normas orientadas a la disminución de la incidencia de las infecciones adquiridas en el ambiente hospitalario.

La resistencia a antimicrobianos de las cepas de $P$. aeruginosa encontradas en pacientes del Hospital Dr. Leonardo Guzmán de Antofagasta, difiere en algunos aspectos respecto de lo reportado en publicaciones nacionales ${ }^{4,15-17,34,40-43}$. Cabe destacar que no existen publicaciones recientes sobre estudios realizados en hospitales de nuestra región que evalúen la susceptibilidad de cepas de $P$. aeruginosa frente a diferentes familias de antimicrobianos.

Lira y cols reportaron un aumento de la resistencia de $P$. aeruginosa frente a amikacina y una disminución de la misma frente a gentamicina, en la década 1987 a 1997, en el Hospital San Juan de Dios de Santiago, llegando a observarse al final del período estudiado una resistencia levemente menor a gentamicina ${ }^{15}$. Lo anterior es concordante con lo encontrado por nosotros, que observamos mayor resistencia de las cepas de $P$. aeruginosa frente a amikacina $(36,8 \%)$ que frente a gentamicina $(26,3 \%)$. Creemos que este cambio del perfil de susceptibilidad se debe al mayor uso actual de la amikacina frente a las infecciones por $P$. aeruginosa y a una menor utilización de la gentamicina en el medio hospitalario local. En 1993 O. Trucco y cols estudiaron 30 cepas de $P$. aeruginosa aisladas desde diferentes hospitales de la Región Metropolitana, encontrando una menor resistencia frente a amikacina $^{41}$. Por otra parte, V. Triantafilo y cols en 1994 estudiaron 68 cepas de $P$. aeruginosa aisladas desde pacientes con infecciones intrahospitalarias, encontrando menor resistencia de las cepas frente a amikacina que frente a gentamicina $^{34}$. En el informe del primer semestre del año 2001 de la red de vigilancia de resistencia antimicrobiana PRONARES, que incluye cepas de $P$. aeruginosa de Iquique, se encontró una resistencia menor a $30 \%$ para gentamicina y bajo $15 \%$ para amikacina ${ }^{42}$. De las quinolonas, la más activa es ciprofloxacina, habiendo nosotros encontrado una mayor resistencia $(68,4 \%$ para ciprofloxacina) de $P$. aeruginosa con respecto a lo reportado en estudios nacionales ${ }^{34,41}$. En el estudio PRONARES, publicado por O. Trucco y cols, también se encontró una alta resistencia $(50 \%)$ a ciprofloxacina en cepas aisladas de pacientes con infecciones nosocomiales ${ }^{42}$. En 1994, Silva y cols encontraron una baja resistencia a ciprofloxacina en 64 cepas de $P$. aeruginosa aisladas en dos centros hospitalarios de Antofagasta ${ }^{43}$; nosotros, por el contrario, observamos una baja susceptibilidad frente a este antimicrobiano. Tales diferencias con respecto a nuestros resultados evidencian cambios importantes en el perfil de susceptibilidad antimicrobiana a quinolonas en el período transcurrido de ocho años. La resistencia de las cepas de $P$. aeruginosa aisladas es marcadamente mayor que la observada en otros trabajos realizados en hospitales del Área Metropolitana ${ }^{34,40}$ y que la reportada por la red PRONARES frente a ceftazidima ${ }^{42}$. En efecto, nosotros encontramos una resistencia de $36,8 \%$ para ceftazidima, mientras que PRONARES informó una resistencia menor a $20 \%{ }^{42}$. Sin embargo, la resistencia encontrada en nuestro estudio $(15,8 \%)$ para cefoperazona/sulbactam es más baja que la encontrada por PRONARES, donde se reporta una resistencia levemente menor a $30 \%{ }^{42}$. Es posible que la baja resistencia a cefoperazona/sulbactam observada en las cepas de Antofagasta se deba a la escasa utilización de este antibacteriano en nuestro medio. La resistencia frente a imipenem fue también considerablemente mayor que la reportada previamente en trabajos nacionales, sobrepasando el $25 \%$ mientras que otros investigadores reportaron una resistencia cercana a $10 \% \%^{34,40,42}$. El período estudiado coincidió con un mayor consumo de imipenem en nuestro centro asistencial, lo cual podría estar relacionado con el hallazgo de esta elevada resistencia al antimicrobiano. Un hallazgo destacable fue que las cepas de $P$. aeruginosa aisladas en nuestro medio fueron altamente sensibles a la combinación de piperacilina/tazobactam, siendo su resistencia de sólo 5,3\%, lo cual está en concordancia con la literatura nacional ${ }^{34}$. Lo anterior podría sugerir que frente a infecciones graves que amenazan la vida del paciente, piperacilina/tazobactam sería una muy buena alternativa terapéutica.

Quedan desafíos futuros en cuanto a corroborar estas altas cifras de resistencia, ya sea por derivación de las cepas resistentes al ISP para confirmación, uso de métodos adicionales, según las recomendaciones actuales o por lo con- 
trario detectar con técnicas de biología molecular la presencia en nuestro hospital de un único clon que esté circulando y que estemos frente a un brote y no de un aumento progresivo de la resistencia.

La elección del antimicrobiano apropiado es de vital importancia en las infecciones graves por $P$. aeruginosa. Conocer a qué antimicrobianos son susceptibles con mayor frecuencia las cepas en cada institución, es fundamental para la elección del tratamiento empírico inicial, ya que los cultivos con estudio de susceptibilidad estarán disponibles recién a las $48-72$ horas de incubación ${ }^{18}$. Por otro lado, un tratamiento acertado puede acortar los tiempos de hospitalización. Nosotros encontramos una baja resistencia a la combinación piperacilina/tazobactam y a cefoperazona/sulbactam, mientras que la resistencia a antimicrobianos de uso frecuente en infecciones por $P$. aeruginosa, como ceftazidima, amikacina y ciprofloxacina, fue mayor a lo reportado por diferentes publicaciones nacionales $15,34,40,42,43$.

Creemos que este estudio puede servir de valiosa referencia para ayudar a la toma de decisiones racionales sobre el uso de los antimicrobianos en la práctica clínica diaria, basadas en un mejor conocimiento de la realidad microbiológica local. Debe ponerse en una balanza el uso de antimicrobianos de costo elevado, a los cuales $P$. aeruginosa es altamente susceptible en nuestro medio, como piperacilina/tazobactam, con la reducción de costos asociados a la duración de la hospitalización y el beneficio para la salud del paciente.

\section{Resumen}

Pseudomonas aeruginosa es un patógeno nosocomial frecuente que presenta elevada resistencia a los antimicrobianos y causa infecciones graves cuando hay alteración de los mecanismos defensivos del paciente. Así, conocer los patrones locales de sensibilidad es importante para la elección del tratamiento antimicrobiano adecuado en cada institución. En este trabajo determinamos la susceptibilidad antimicrobiana de cepas de $P$. aeruginosa aisladas desde pacientes atendidos en el Hospital Regional de Antofagasta. La mayoría de los pacientes tenía alguna condición predisponente a la infección y $48 \%$ tenía una infección grave. Las cepas mostraron mayor resistencia a los antimicrobianos que lo reportado en trabajos nacionales previos. Las cepas fueron altamente resistentes a amikacina $(36,8 \%)$, ceftazidima $(36,8 \%)$ y ciprofloxacina $(68,4 \%)$, moderadamente resistentes a imipenem (26,3\%), mientras que eran escasamente resistentes a piperacilina/tazobactam $(5,3 \%)$ y cefoperazona/sulbactam $(15,8 \%)$, Este es el primer trabajo, realizado en nuestra región, que estudia la susceptibilidad de $P$. aeruginosa frente a distintos grupos de antimicrobianos utilizados en clínica.

\section{Agradecimientos}

Se agradece la valiosa colaboración de Juan Pastenes y Nelson Labarca del Laboratorio de Microbiología de la U. de Antofagasta, y de Carmen Chambel del Laboratorio del Hospital Regional de Antofagasta.

\section{Bibliografía}

1.- Van Eldere J. Multicentre surveillance of Pseudomonas aeruginosa susceptibility patterns in nosocomial infections. J Antimicrob Chemother 2003; 51: 347 52.

2.- Assadian O, Apfalter P, Assadian A, Makristathis A, Daxboeck F, Koller W et al. Antimicrobial susceptibility profiles of clinically relevant blood culture isolates from nine surgical intensive care units. Eur J Clin Microbiol Infect Dis 2002; 21: 743-7.

3.- Gordon S, Serkey J, Keys T, Ryan T, Fatica C, Schmitt $\mathrm{S}$ et al. Secular trends in nosocomial bloodstream infections in a 55-bed cardiothoracic intensive care unit. Ann Thorac Surg 1998; 65: 95-100.

4.- Escobar M, González L, Álvarez M, Álvarez P, Pinto M. Infecciones intrahospitalarias en una unidad de cuidados intensivos. Estudios prospectivos de algunos aspectos bacteriológicos y epidemiológicos. Bol Hosp SJ de Dios 1987; 34: 8-13.

5.- Subiabre D, Gálvez S, Ossandón B. Neumonía nosocomial en pacientes en ventilación mecánica. Bol Hosp Viña del Mar 1994; 50: 174-9.

6.- Brenner P, Pohlenz M, Otaíza F, Valenzuela M. Informe de vigilancia epidemiológica de las infecciones intrahospitalarias. Ministerio de Salud de Chile. 2001.

7.- Todar's Online Textbook of Bacteriology. University of Wisconsin-Madison Department of Bacteriology. www.textbookofmicrobiology.net/Pseudomonas.html

8.- Kluytmans J. Surgical infections including burns. In: Wenzel RP, editor. Prevention and Control of Nosocomial Infections. $3^{\text {rd }}$. ed. Baltimore Williams \& Wilkins, 1997; 841-65.

9.- Jones A, Dodd M, Doherty C, Govan J, Webb A. Increased treatment requirements of patients with cystic fibrosis who harbour highly transmissible strain of Pseudomonas aeruginosa. Thorax 2002; 57: 924-5.

10.- Van Delden C, Iglewski B. Cell-to-cell signaling and Pseudomonas aeruginosa infections. Emerg Infect Dis 1998; 4: 551.

11.- Brooks G, Morse S, Butel J. Pseudomonas. En: Jawetz, Melnick y Adelberg Microbiología Médica. 16 a ed. México D.F., El Manual Moderno 1999; 699-701.

12.- Pollack M, Ohl C. Infections due to Pseudomonas species and related microorganisms. In: Harrison's Principles of Internal Medicine. $15^{\text {th }}$ ed. Mc GrawHill, U.S.A. 2002; 1138-45.

13.- Hoffken G, Niederman M. Nosocomial pneumonia: the importance of a de-escalating strategy for antibiotic treatment of pneumonia in the ICU. Chest 2002; 122: 2183-96.

14.- De Freitas A, Barth A. Antibiotic resistance and molecular typing of Pseudomonas aeruginosa: focus on imipenem. Braz J Infect Dis 2002; 6: 1-7. 
15.- Lira G, Giglio M, Zúñiga M, Pinto M. Consumo de antimicrobianos y variación de resistencia de bacilos Gram negativos en un período de diez años. Hospital San Juan de Dios. Rev Chil Infect 1999; 16: 199-210.

16.- Beltrán C, Thompson L, Borie C, González C, Silva M. Alta resistencia a ceftazidima en bacilos Gram negativos intrahospitalarios. Rev Chil Infect 1995; 12: $129-35$.

17.- Zemelman R, Bello H, Domínguez M, González G, Mella S, García A. Activity of imipenem, third generation cephalosporins, aztreonam and ciprofloxacin against multiresistant Gram-negative bacilli isolated from Chilean hospitals. J Antimicrob Chemother 1993; 32: 413-9.

18.- Procedimientos y técnicas de laboratorio. Instituto de Salud Pública. Ministerio de Salud 2001; Chile.

19.- ERICCSON H, SHERRIS J. Antibiotic sensibility testing: report of an international collaborative study. Act Pathol Microbiol Scand, Sect B 1971; 217 (Suppl): S1-S90.

20.- National Committee for Clinical Laboratory Standards (NCCLS). Scheme for preparing dilutions of antimicrobial agents to be used in agar dilution susceptibility tests. NCCLS document M7-A6. 2003.

21.- National Committee for Clinical Laboratory Standards (NCCLS). 2003. Minimum inhibitory concentration (MIC) interpretative standards $(\mu \mathrm{g} / \mathrm{ml})$ for Pseudomonas aeruginosa and other non-Enterobacteriaceae. NCCLS document M7-A6.

22.- Laboratorios Pfizer. Productos. Sulperazon. www. pfizer.com.ar

23.- Gencer S, Ak O, Benzonana N, Batirel A, Ozer S. Susceptibility patterns and cross resistance of antibiotics against Pseudomonas aeruginosa in a teaching hospital of Turkey. Ann Clin Microbiol Antimicrob 2002; Oct 9.

24.- National Committee for Clinical Laboratory Standards (NCCLS). 2003. Acceptable quality control ranges of minimum inhibitory concentration (MICs) $(\mu \mathrm{g} / \mathrm{ml})$ for reference strains. NCCLS document M7-A6.

25.- Dunn M, Wunderink R. Ventilator-associated pneumonia caused by Pseudomonas infection. Clin Chest Medicine 1995; 16: 95-109.

26.- Wiblin R. Nosocomial pneumonia. In Wenzel R, Prevention and Control of Nosocomial Infections. $3^{\text {rd }}$. ed. Baltimore, Williams \& Wilkins, 1997; 807-19.

27.- Fridkin S, Hill H, Volkova N, Edwards J, Lawton R, Gaynes $\mathrm{R}$ et al. Temporal changes in prevalence of antimicrobial resistance in 23 US hospitals. Emerg Infect Dis 2002; 8: 697-701.

28.- Allegranzi B, Luzzati R, Luzzani A, Girardini F, Antozzi $\mathrm{L}$, Raiteri $\mathrm{R}$ et al. Impact of antibiotic changes in empirical therapy on antimicrobial resistance in intensive care unit-acquired infections. J Hosp Infect 2002; 52: 136-40.

29.- Kaempffer A, Sepúlveda L, Vargas R, Nocera C, Oyarzo M, Nuñez E. La infección intrahospitalaria: sus costos y probable asociación con las condiciones para la eficiencia. Cuad Méd Soc 1988; 29: 93-8.

30.- Otaíza F, Pinto M, Rodríguez H, Lobos H, Peake G.
Situación nacional de infecciones intrahospitalarias en Chile. Rev Méd Chile 1987; 115: 74-80.

31.- Río Y, Pina P, Jurin F, Allouch P, Didion J, Chardon $\mathrm{H}$ et al. Susceptibility of Pseudomonas aeruginosa to antibiotics isolated from patients of care units in France in 1998. Resistant phenotypes to betalactams. Pathol Biol 2002; 50: 12-7.

32.- Poirel L, Weldhagen G, De Champs C, Nordmann P. A nosocomial outbreaks of Pseudomonas aeruginosa isolates expressing the extended-spectrum $\beta$-lactamase GES-2 in South Africa. J Antimicrob Chemother 2002; 49: $561-5$.

33.- Harris A, Perencevich E, Roghmann M, Morris G, Kaye K, Johnson J. Risk factors for piperacillintazobactam-resistant Pseudomonas aeruginosa among hospitalized patients. Antimicrob Agents Chemother 2002; 46: 854-8.

34.- Triantafilo V, Fica A, Silva M, Thompson L. E-test para determinar concentraciones inhibitorias mínimas, estimar la diversidad bacteriana e identificar presuntivamente $\beta$-lactamasas en cepas de Pseudomonas aeruginosa y Acinetobacter baumannii asociadas a infecciones intrahospitalarias. Rev Méd Chile 1997; 125: 149-60.

35.- Simpson A, Suputtamongkol Y, Smith M, Angus B, Rajanuwong A, Wuthiekanun V et al. Comparison of imipenem and ceftazidime as therapy for severe melioidosis. Clin Infect Dis 1999; 29: 381-7.

36.- Mendelson M, Gurtman A, Szabo S, Neibart E, Meyers B, Policar M et al. Pseudomonas aeruginosa bacteremia in patients with AIDS. Clin Infect Dis 1994; 18 : 886-95.

37.- Nakajima A, Sugimoto Y, Yoneyama H, Nakae T. High level fluoroquinolone resistance in Pseudomonas aeruginosa due to interplay of the MexAB-OprM efflux pump and the DNA gyrase mutation. Microbiol Immunol 2002; 46: 391-5.

38.- Shepp D, Tang I, Ramundo M, Kaplan M. Serious Pseudomonas aeruginosa infection in AIDS. J Acquir Immun Defic Syndr 1994; 7: 823-31.

39.- Carmeli Y, Troillet N, Karchmer A, Samore M. Health and economic outcomes of antibiotic resistance in Pseudomonas aeruginosa. Arch Intern Med 1999; 159: 1127-32.

40.- Basualdo W, Prado V, Ojeda O. Situación actual de la actividad in vitro de ceftazidima y cefuroxima en relación a otros antimicrobianos, frente a patógenos Gram positivo y negativo. Rev Chil Infect 1993; 10: 175-85.

41.- Trucco O, Prado V, Arellano C. Actividad in vitro de fleroxacina en relación a otros 9 antimicrobianos de uso habitual, frente a patógenos frecuentes en patologías urinarias, entéricas y sistémicas. Rev Chil Infect 1993; 10: 115-24.

42.- Trucco O, Prado V, Duran C, Grupo Pronares. Red de vigilancia de resistencia antimicrobiana PRONARES. Informe primer semestre 2001. Rev Chil Infect 2002; 19 (S2):140-8.

43.- Silva J, Herrera N, Prado V. Actividad comparativa in vitro de 6 fluoroquinolonas sobre bacterias patógenas de origen clínico. Rev Chil Infect 1995; 12: 40-7.

Correspondencia a:

Alcides Zambrano Fernández

E-mail: alcidedu@yahoo.es 